International Journal of Modern Physics A

(C) World Scientific Publishing Company

\title{
CRITICAL BEHAVIOUR IN STATISTICAL QCD
}

\author{
HELMUT SATZ* \\ Department of Physics, Bielefeld University, Universitätsstrasse 25 \\ D-33615 Bielefeld, Germany \\ Received (Day Month Year) \\ Revised (Day Month Year)
}

\begin{abstract}
We survey the critical behaviour of strongly interacting matter, as determined in finite temperature lattice QCD.

Keywords: 05.70.Fh; 12.38.Mh.
\end{abstract}

\section{Phases of Strongly Interacting Matter}

What happens to strongly interacting matter in the limit of high temperature and/or density? This question has fascinated physicists ever since short range strong interactions and the resulting multiple hadron production were discovered. Let us look at some of the features that have emerged.

- Hadrons have an intrinsic size, with a radius $r_{h} \simeq 1 \mathrm{fm}$, and hence a hadron needs a space of volume $V_{h} \simeq(4 \pi / 3) r_{h}^{3}$ in order to exist. This suggests a limiting density $n_{c}$ of hadronic mattel 1 , with $n_{c}=1 / V_{h} \simeq 1.5 n_{0}$, where $n_{0} \simeq 0.17 \mathrm{fm}^{-3}$ denotes the density of normal nuclear matter.

- Hadronic interactions provide abundant resonance production, and the resulting number $\rho(m)$ of hadron species increases exponentially as function of the resonance mass $m, \rho(m) \sim \exp (b m)$. Such a form for $\rho(m)$ appeared first in the statistical bootstrap model, based on self-similar resonance formation or decay 2. It was then also obtained in the more dynamical dual resonance approach 3 . In hadron thermodynamics, the exponential increase of the resonance degeneracy results in an upper limit for the temperature of hadronic matter $\stackrel{2}{2}, T_{c}=1 / b \simeq 150-200 \mathrm{MeV}$.

- What happens beyond $T_{c}$ ? In QCD, hadrons are dimensionful color-neutral bound states of more basic pointlike colored quarks and gluons. Hadronic matter, consisting of colorless constituents of hadronic dimensions, can therefore turn at high temperatures and/or densities into a quark-gluon

*also at CFIF, Instituto Superior Técnico, Lisbon, Portugal. 
plasma of pointlike colored quarks and gluons as constituents 4 . This deconfinement transition leads to a colour-conducting state and thus is the QCD counterpart of the insulator-conductor transition in atomic matter

- A shift in the effective constituent mass is a second transition phenomenon expected from the behavior of atomic matter. At $T=0$, in vacuum, quarks dress themselves with gluons to form the constituent quarks that make up hadrons. As a result, the bare quark mass $m_{q} \sim 0$ is replaced by a constituent quark mass $M_{q} \sim 300 \mathrm{MeV}$. In a hot medium, this dressing melts and $M_{q} \rightarrow 0$. Since the QCD Lagrangian for $m_{q}=0$ is chirally symmetric, $M_{q} \neq 0$ implies spontaneous chiral symmetry breaking. The melting $M_{q} \rightarrow 0$ thus corresponds to chiral symmetry restoration. We shall see later on that in QCD, as in atomic physics, the shift of the constituent mass coincides with the onset of conductivity.

- A third type of transition would set in if the attractive interaction between quarks leads in the deconfined phase to the formation of colored bosonic diquark pairs, the Cooper pairs of QCD. These diquarks can then condense at low temperature to form a color superconductor. Heating will dissociate the diquark pairs and turn the color superconductor into a normal color conductor.

Using the baryochemical potential $\mu$ as a measure for the baryon density of the system, we thus expect the phase diagram of QCD to have the schematic form shown in Fig. 1 Given QCD as the fundamental theory of strong interactions, we can use the QCD Lagrangian as dynamics input to derive the resulting thermodynamics of strongly interacting matter. For vanishing baryochemical potential, $\mu=0$, this can be evaluated with the help of the lattice regularisation, leading to finite temperature lattice QCD.

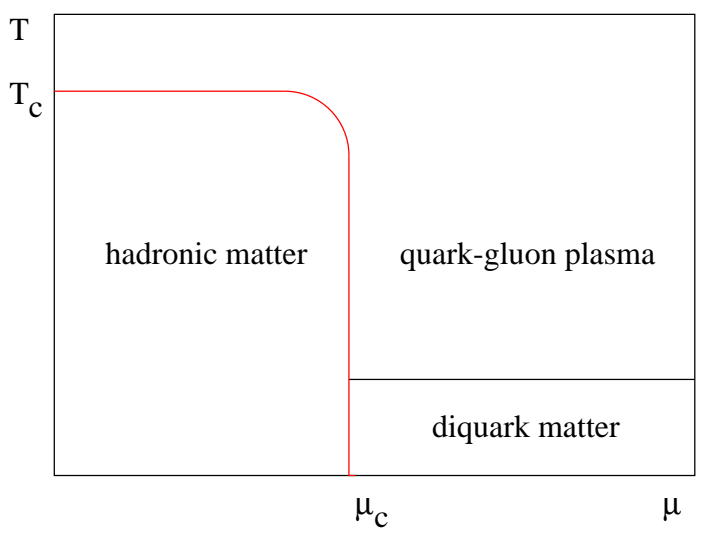

Fig. 1. The phase diagram of QCD 


\section{Finite Temperature Lattice QCD}

QCD is defined by the Lagrangian

$$
\mathcal{L}=-\frac{1}{4} F_{\mu \nu}^{a} F_{a}^{\mu \nu}-\sum_{f} \bar{\psi}_{\alpha}^{f}\left(i \gamma^{\mu} \partial_{\mu}+m_{f}-g \gamma^{\mu} A_{\mu}\right)^{\alpha \beta} \psi_{\beta}^{f},
$$

with

$$
F_{\mu \nu}^{a}=\left(\partial_{\mu} A_{\nu}^{a}-\partial_{\nu} A_{\mu}^{a}-g f_{b c}^{a} A_{\mu}^{b} A_{\nu}^{c}\right) .
$$

Here $A_{\mu}^{a}$ denotes the gluon field of colour $a(a=1,2, \ldots, 8)$ and $\psi_{\alpha}^{f}$ the quark field of colour $\alpha(\alpha=1,2,3)$ and flavour $f$; the input ('bare') quark masses are given by $m_{f}$. With the dynamics thus determined, the corresponding thermodynamics is obtained from the partition function, which is most suitably expressed as a functional path integral,

$$
Z(T, V)=\int d A d \psi d \bar{\psi} \exp \left(-\int_{V} d^{3} x \int_{0}^{1 / T} d \tau \mathcal{L}(A, \psi, \bar{\psi})\right),
$$

since this form involves directly the Lagrangian density defining the theory. The spatial integration in the exponent of Eq. (3) is performed over the entire spatial volume $V$ of the system; in the thermodynamic limit it becomes infinite. The time component $x_{0}$ is "rotated" to become purely imaginary, $\tau=i x_{0}$, thus turning the Minkowski manifold, on which the fields $A$ and $\psi$ are originally defined, into a Euclidean space. The integration over $\tau$ in Eq. (3) runs over a finite slice whose thickness is determined by the temperature of the system.

From $Z(T, V)$, all thermodynamical observables can be calculated in the usual fashion. Thus

$$
\epsilon=\left(T^{2} / V\right)\left(\frac{\partial \ln Z}{\partial T}\right)_{V}
$$

gives the energy density, and

$$
P=T\left(\frac{\partial \ln Z}{\partial V}\right)_{T}
$$

the pressure. For the study of critical behaviour, long range correlations and multiparticle interactions are of crucial importance; hence perturbation theory cannot be used. The necessary non-perturbative regularisation scheme is provided by the lattice formulation of $\mathrm{QCD}^{7}$; ; it leads to a form which can be evaluated numerically by computer simulation $[8$.

The first variable to consider is the deconfinement measure given by the Polyakov loop 1011

$$
L(T) \sim \lim _{r \rightarrow \infty} \exp \{-V(r) / T\}
$$

where $V(r)$ is the potential between a static quark-antiquark pair separated by a distance $r$. In the limit of large input quark mass, $V(\infty)=\infty$ in the confined phase, 
so that then $L=0$. Colour screening, on the other hand, makes $V(r)$ finite at large $r$, so that in the deconfined phase, $L$ does not vanish. It thus becomes an 'order parameter' like the magnetisation in the Ising model. In the large quark mass limit, QCD reduces to pure $S U(3)$ gauge theory, which is invariant under a global $Z_{3}$ symmetry. The Polyakov loop provides a measure of the state of the system under this symmetry: it vanishes for $Z_{3}$ symmetric states and becomes finite when $Z_{3}$ is spontaneously broken. Hence the critical behaviour of $S U(3)$ gauge theory is in the same universality class as that of $Z_{3}$ spin theory (the 3-state Potts model): both are due to the spontaneous symmetry breaking of a global $Z_{3}$ symmetry 12 .

For finite quark mass $m_{q}, V(r)$ remains finite for $r \rightarrow \infty$, since the 'string' between the two colour charges 'breaks' when the corresponding potential energy becomes equal to the mass $M_{h}$ of the lowest hadron; beyond this point, it becomes energetically more favourable to produce an additional hadron. Hence now $L$ no longer vanishes in the confined phase, but only becomes exponentially small there,

$$
L(T) \sim \exp \left\{-M_{h} / T\right\}
$$

here $M_{h}$ is of the order of the $\rho$-mass, so that $L \sim 10^{-2}$, rather than zero. Deconfinement is thus indeed much like the insulator-conductor transition, for which the order parameter, the conductivity $\sigma(T)$, also does not really vanish for $T>0$, but with $\sigma(T) \sim \exp \{-\Delta E / T\}$ is only exponentially small, since thermal ionisation (with ionisation energy $\Delta E$ ) produces a small number of unbound electrons even in the insulator phase.

Fig. 2] a shows recent lattice result $\$ 13$ for $L(T)$ and the corresponding susceptibility $\chi_{L}(T) \sim\left\langle L^{2}\right\rangle-\langle L\rangle^{2}$. The calculations were performed for the case of two flavours of light quarks, using a current quark mass about four times larger than that needed for the physical pion mass. We note that $L(T)$ undergoes the expected sudden increase from a small confinement to a much larger deconfinement value. The sharp peak of $\chi_{L}(T)$ defines quite well the transition temperature $T_{c}$, which we shall shortly specify in physical units.

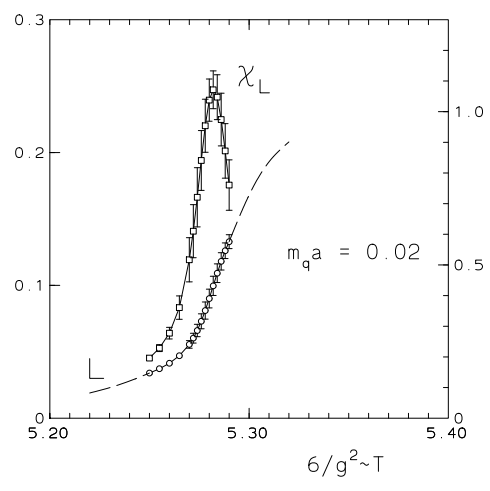

(a)

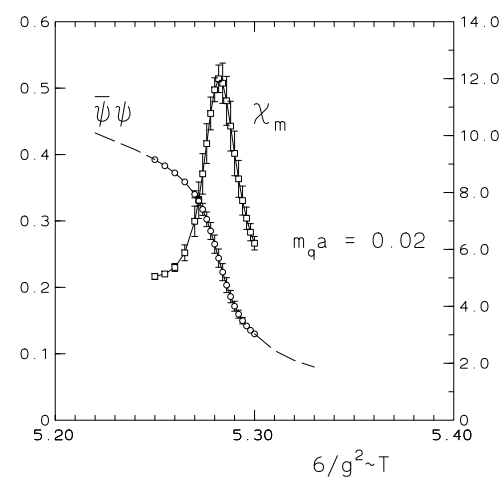

(b)

Fig. 2. Polyakov loop and chiral condensate in two-flavour QCD 13 
The next quantity to consider is the effective quark mass; it is measured by the expectation value of the corresponding term in the Lagrangian, $\langle\bar{\psi} \psi\rangle(T)$. In the limit of vanishing current quark mass, the Lagrangian becomes chirally symmetric and $\langle\bar{\psi} \psi\rangle(T)$ the corresponding order parameter. In the confined phase, with effective constituent quark masses $M_{q} \simeq 0.3 \mathrm{GeV}$, this chiral symmetry is spontaneously broken, while in the deconfined phase, at high enough temperature, we expect its restoration. In the real world, with finite pion and hence finite current quark mass, this symmetry is also only approximate, since $\langle\bar{\psi} \psi\rangle(T)$ now never vanishes at finite $T$.

The behaviour of $\langle\bar{\psi} \psi\rangle(T)$ and the corresponding susceptibility $\chi_{m} \sim$ $\partial\langle\bar{\psi} \psi\rangle / \partial m_{q}$ are shown in Fig. 2] $\mathrm{b} \frac{13}{13}$, calculated for the same case as above in Fig. 2 a. We note here the expected sudden drop of the effective quark mass and the associated sharp peak in the susceptibility. The temperature at which this occurs coincides with that obtained through the deconfinement measure. We therefore conclude that at vanishing baryon number density, quark deconfinement and the shift from constituent to current quark mass coincide. We thus obtain for $\mu_{B}=0 \mathrm{a}$ rather well defined phase structure, consisting of a confined phase for $T<T_{c}$, with $L(T) \simeq 0$ and $\langle\bar{\psi} \psi\rangle(T) \neq 0$, and a deconfined phase for $T>T_{c}$ with $L(T) \neq 0$ and $\langle\bar{\psi} \psi\rangle(T) \simeq 0$. The underlying symmetries associated to the critical behaviour at $T=T_{c}$, the $Z_{3}$ symmetry of deconfinement and the chiral symmetry of the quark mass shift, become exact in the limits $m_{q} \rightarrow \infty$ and $m_{q} \rightarrow 0$, respectively. In the real world, both symmetries are only approximate; nevertheless, we see from Fig. 2 that both associated measures retain an almost critical behaviour.

Next we come to the behaviour of energy density $\epsilon$ and pressure $P$ at deconfinement. In Fig. 3. it is seen that $\epsilon / T^{4}$ changes quite abruptly at the above determined critical temperature $T=T_{c}$, increasing from a low hadronic value to one slightly below that expected for an ideal gas of massless quarks and gluons 14 .

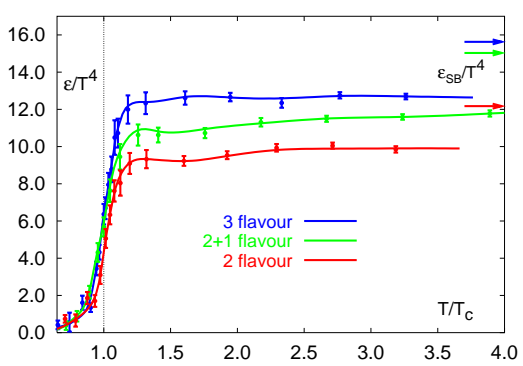

Fig. 3. Energy density vs. temperature 14

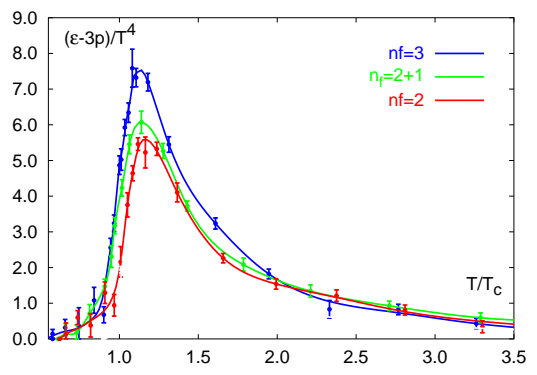

Fig. 4. Interaction measure vs. temperature 15

Besides the sudden increase at deconfinement, there are two further points to note. In the region $T_{c}<T<2 T_{c}$, there still remain strong interaction effects. As seen in Fig. [4 the 'interaction measure' $\Delta=(\epsilon-3 P) / T^{4}$ remains sizeable and does not vanish, as it would for an ideal gas of massless constituents 15 . In the simple model 
of the previous section, such an effect arose due to the bag pressure, and in actual QCD, one can also interpret it in such a fashion 16. It has also been considered in terms of a gradual onset of deconfinement starting from high momenta 17 , and most recently as a possible consequence of coloured "resonance" states 18 . The second point to note is that the thermodynamic observables remain about $10 \%$ below their Stefan-Boltzmann values (marked "SB" in Fig. 3) even at very high temperatures, where the interaction measure becomes very small. Such deviations from ideal gas behaviour can be expressed to a large extent in terms of effective 'thermal' masses $m_{\text {th }}$ of quarks and gluons, with $m_{\text {th }} \sim g(T) T 192021$. Maintaining the next-toleading order term in mass in the Stefan-Boltzmann form gives for the pressure

$$
P=c T^{4}\left[1-a\left(\frac{m_{\mathrm{th}}}{T}\right)^{2}\right]=c T^{4}\left[1-a g^{2}(T)\right]
$$

and for the energy density

$$
\begin{aligned}
\epsilon & =3 c T^{4}\left[1-\frac{a}{3}\left(\frac{m_{\mathrm{th}}}{T}\right)^{2}-\frac{2 a}{3}\left(\frac{m_{\mathrm{th}}}{T}\right)\left(\frac{d m_{\mathrm{th}}}{d T}\right)\right] \\
& =3 c T^{4}\left[1-a g^{2}(T)+\frac{2 a m_{\mathrm{th}}}{3}\left(\frac{d g}{d T}\right)\right],
\end{aligned}
$$

where $c$ and $a$ are colour- and flavour-dependent positive constants. The deviations of $P$ and $\epsilon$ from the massless Stefan-Boltzmann form thus vanish only logarithmically, with $g(T) \sim 1 / \ln T$, while the interaction measure

$$
\Delta=2 \operatorname{acg}(T) T \frac{d g}{d T}
$$

vanishes up to terms of order $T d g / d T$.

Finally we turn to the value of the transition temperature. Since QCD (in the limit of massless quarks) does not contain any dimensional parameters, $T_{c}$ can only be obtained in physical units by expressing it in terms of some other known observable which can also be calculated on the lattice, such as the $\rho$-mass, the proton mass, or the string tension. In the continuum limit, all different ways should lead to the same result. Within the present accuracy, they define the uncertainty so far still inherent in the lattice evaluation of QCD. Using the $\rho$-mass to fix the scale leads to $T_{c} \simeq 0.15 \mathrm{GeV}$, while the string tension still allows values as large as $T_{c} \simeq 0.20 \mathrm{GeV}$. This means that energy densities of some $1-2 \mathrm{GeV} / \mathrm{fm}^{3}$ are needed in order to produce a medium of deconfined quarks and gluons.

In summary, finite temperature lattice QCD at vanishing baryon density shows

- that there is a transition leading to colour deconfinement coincident with chiral symmetry restoration at $T_{c} \simeq 0.15-0.20 \mathrm{GeV}$;

- that this transition is accompanied by a sudden increase in the energy density (the "latent heat of deconfinement") from a small hadronic value to a much larger value, about $10 \%$ below that of an ideal quark-gluon plasma. 
In the following section, we want to address in more detail the nature of the critical behaviour encountered at the transition.

\section{The Nature of the Transition}

We begin with the behaviour for vanishing baryon density $(\mu=0)$ and come to $\mu \neq 0$ at the end. Consider the case of three quark species, $u, d, s$.

- In the limit $m_{q} \rightarrow \infty$ for all quark species, we recover pure $S U(3)$ gauge theory, with a deconfinement phase transition provided by spontaneous $Z_{3}$ breaking. It is first order, as is the case for the corresponding spin system, the 3 -state Potts model.

- For $m_{q} \rightarrow 0$ for all quark masses, the Lagrangian becomes chirally symmetric, so that we have a phase transition corresponding to chiral symmetry restoration. In the case of three massless quarks, the transition is also of first order.

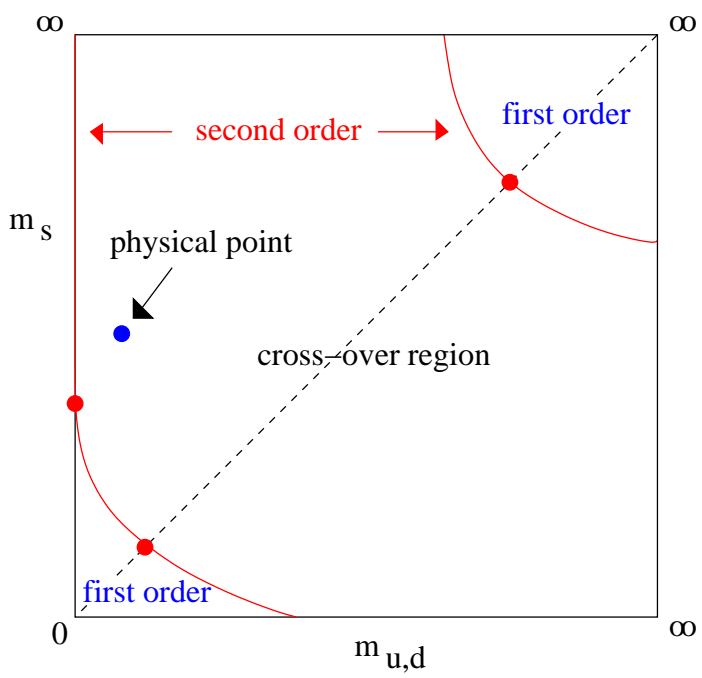

Fig. 5. The nature of thermal critical behaviour in QCD

- For $0<m_{q}<\infty$, there is neither spontaneous $Z_{3}$ breaking nor chiral symmetry restoration. Hence in general, there is no singular behaviour, apart from the transient disappearence of the first order discontinuities on a line of second order transitions. Beyond this, there is no genuine phase transition, but only a "rapid cross-over" from confinement to deconfinement. The overall behaviour is summarized in Fig. [5

- As already implicitely noted above, both "order parameters" $L(T)$ and $\chi(T)$ nevertheless show a sharp temperature variation for all values of $m_{q}$, 
so that it is in fact possible to define quite well a common cross-over point $T_{c}$.

- The nature of the transition thus depends quite sensitively on the number of flavours $N_{f}$ and the quark mass values: it can be a genuine phase transition (first order or continuous), or just a rapid cross-over. The case realized in nature, the "physical point", corresponds to small $u, d$ masses and a larger $s$-quark mass. It is fairly certain today that this point falls into the cross-over region.

- Finally we consider briefly the case of finite baryon density, $\mu \neq 022$. Here the conventional computer algorithms of lattice QCD break down, and hence new calculation methods have to be developed. First such attempts (reweighting 23, analytic continuation 25, power series 26 ) suggest the phase diagram shown in Fig. 6 The position of the critical end-point of the line of first order transition depends on the position of the physical point in the

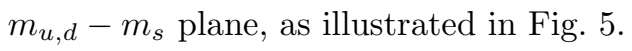

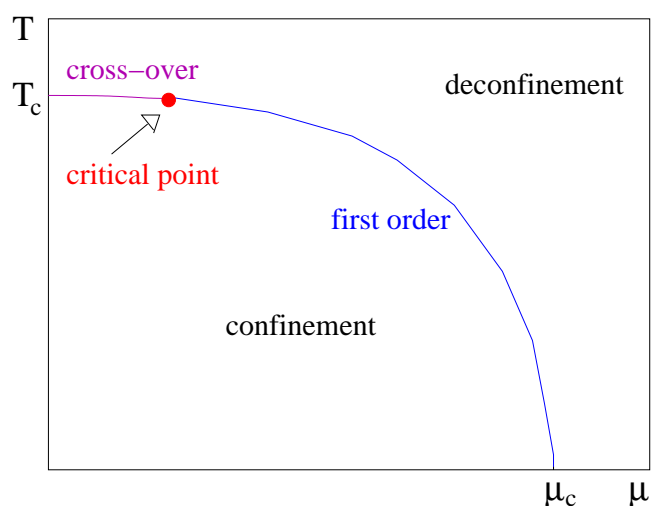

Fig. 6. Deconfinement in the $T, \mu$ plane

The critical behaviour for strongly interacting matter at low or vanishing baryon density, describing the onset of confinement in the early universe and in high energy nuclear collisions, thus occurs in the rather enigmatic form of a "rapid cross-over". There is no thermal singularity and hence, in a strict sense, there are neither distinct states of matter nor phase transitions between them. So what does the often mentioned experimental search for a "new state of matter" really mean? How can a new state appear without a phase transition? Is there a more general way to define and distinguish different states of bulk media? After all, in statistical QCD one does find that thermodynamic observables - energy and entropy densities, pressure, as well as the "order parameters" $L(T)$ and $\chi(T)$ - continue to change rapidly and thus define a rather clear transition line in the entire cross-over region. Why is this so, what is the mechanism which causes such a transition? 
In closing this section, we consider a speculative answer to this rather fundamental question. It is known that in spin systems, when the partition function becomes analytic and hence there is no more thermal critical behaviour, the onset of large cluster formation, i.e., percolation, persists as a geometric form of critical behaviour persits 28. This can be applied also to the question of deconfinement 29 . Consider hadrons of intrinsic size $V_{h}=(4 \pi / 3) r_{h}^{3}$, with $r_{h} \simeq 0.8 \mathrm{fm}$. In three-dimensional space, the formation of a connected large-scale cluster first occurs at the density

$$
n_{c}=\frac{0.34}{V_{h}} \simeq 0.16 \mathrm{fm}^{-3} \text {. }
$$

This point specifies the onset of hadronic matter, in contrast to a gas of hadrons, and it indeed correctly reproduces the density of normal nuclear matter. However, at this density the vacuum as connected medium also still exists (see Fig. $7 \mathrm{~h}$ ).

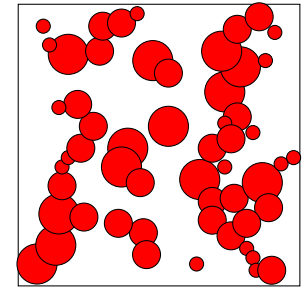

(a)

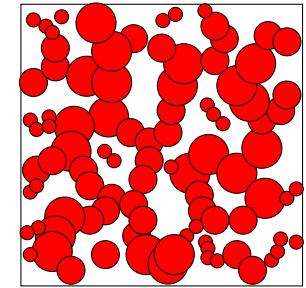

(b)

Fig. 7. Hadron and vacuum percolation thresholds

To prevent infinite connecting vacuum clusters, a much higher hadron density is needed. Measured in hadronic size units, the vacuum disappears for

$$
\bar{n}_{c}=\frac{1.24}{V_{h}} \simeq 0.56 \mathrm{fm}^{-3},
$$

schematically illustrated in Fig. 17b. If we assume that at this point, the medium is of an ideal gas of all known hadrons and hadronic resonances, then we can calculate the temperature of the gas at the density $\bar{n}_{c}: n_{\text {res }}\left(T_{c}\right)=\bar{n}_{c}$ implies $T_{c} \simeq 170 \mathrm{MeV}$, which agrees with the value of the deconfinement temperature found in lattice QCD for $\mu=0$.

We can thus use percolation to define the states of hadronic matter. At low density, we have a hadron gas, which at the percolation point $n_{c}$ turns into connected hadronic matter. When this becomes so dense that only isolated vacuum bubbles survive, at $\bar{n}_{c}$, it turns into a quark-gluon plasma. This approach provides the correct values both for the density of standard nuclear matter and for the deconfinement transition temperature.

Such considerations may in fact well be of a more general nature than the problem of states and transitions in strong interaction physics. The question of whether 
symmetry or connectivity (cluster formation) determines the different states of many-body systems has intrigued theorists in statistical physics for quite a long time 27 . The lesson learned from spin systems appears to be that cluster formation and the associated critical behaviour are the more general feature, which under certain conditions can also lead to thermal criticality, i.e., singular behaviour of the partition function.

\section{References}

1. I. Ya. Pomeranchuk, Doklady Akad. Nauk. SSSR 78

2. R. Hagedorn, Nuovo Cim. Suppl. 3 (1965) 147;

Nuovo Cim. 56A (1968) 1027.

3. K. Bardakci and S. Mandelstam, Phys. Rev. 184 (1969) 1640;

S. Fubini and G. Veneziano, Nuovo Cim. 64A (1969) 811.

4. N. Cabbibbo and G. Parisi, Phys. Lett. 59 B (1975) 67.

5. H. Satz, Fortsch. Physik 33 (1985) 259.

6. M. Asakawa and T. Hatsuda, Nucl. Phys. A 610 (1996) 470c.

7. K. Wilson, Phys. Rev. D10 (1974) 2445.

8. M. Creutz, Phys. Rev. D 21 (1980) 2308.

9. F. Karsch and E. Laermann, in Quark-Gluon Plasma III, R./ C./ Hwa (Ed.), World Scientific, Singapore 2004.

10. L. D. McLerran and B. Svetitsky, Phys. Lett. 98 B (1981) 195 and Phys. Rev. D 24 (1981) 450.

11. J. Kuti, J. Polónyi and K. Szlachányi, Phys. Lett. 98B (1981) 199.

12. B. Svetitsky and L. G. Yaffe, Nucl. Phys. B 210 [FS6] (1982) 423.

13. F. Karsch and E. Laermann, Phys. Rev. D 50 (1994) 6954.

14. F. Karsch, E. Laermann and A. Peikert, Phys. Lett. B 478 (2000) 447;

F. Karsch, Lect. Notes Phys. 583 (2002) 209.

15. A. Peikert, F. Karsch and E. Laermann, Nucl. Phys. B 83-84 (2000) 390.

16. M. Asakawa and T. Hatsuda, Nucl. Phys. A 610 (1996) 470c.

17. J. Engels et al., Z. Phys. C 42 (1989) 341.

18. E. Shuryak and I. Zahed, Phys. Rev. C 70 (2004) 021901.

19. J. Engels et al., Z. Phys. C 42 (1989) 341.

20. V. Goloviznin and H. Satz, Z. Phys. C 57 (1993) 671.

21. F. Karsch, A. Patkos and P. Petreczky, Phys. Lett. B 401 (1997) 69.

22. M. A. Halasz et al., Phys. Rev. D 58 (1998) 096007.

23. Z. Fodor and S. Katz, JHEP 0203 (2002) 014.

24. P. de Forcrand and O. Philipsen, Nucl. Phys. B 642 (2002) 290.

25. M.-P. Lombardo, Phys. Rev. D 67 (2003) 014505.

26. C. R. Allton et al., Phys. Rev. D 68 (2003) 014507.

27. M. E. Fisher, Physica 3 (1967) 255.

28. H. Satz, Nucl. Phys. A 642 (1998) 130c.

29. V. Magas and H. Satz, Europ. Phys. J. C 32 (2003) 115. 